\title{
Estudio sobre los factores de diseño en un producto mediante análisis de componentes principales
}

\author{
Agudo-Vicente, Begoña; Hernandis-Ortuño, Bernabé; Agustín-Fonfria, Miguel Ángel \& Esnal- \\ Angulo, Iñaki. \\ ${ }^{a}$ PhD candidate, Universitat Politècnica de Valencia, Spain. bego.agudo.vicente@gmail.com. \\ ${ }^{\mathrm{b}} \mathrm{PhD}$, Full Professor, Universitat Politècnica de València, Spain. bhernand@degi.upv.es. \\ ${ }^{\mathrm{c}}$ MSc, Full professor, Universitat Politècnica de Valencia, Spain. magustin@upv.es. \\ ${ }^{\mathrm{d}} \mathrm{PhD}$ candidate, Universitat Politècnica de Valencia, Spain. iesan@doctor.upv.es.
}

\begin{abstract}
Resumen
Se pretende determinar el grado de importancia de los factores a considerar en el diseño de un producto, a partir de las diferentes consideraciones contempladas, desde la perspectiva del consumidor y del diseñador. Mediante la aplicación del método de Análisis de Componentes Principales, analizamos a partir de esta investigación y mediante el estudio de un caso, los atributos deseables para un producto, según la opinión de expertos y consumidores. Se comprueba tras la aplicación del método la existencia de subsistemas que explican la necesidad de considerar determinados atributos (variables del sistema), teniendo en cuenta su grado de participación y afinidad en subsistemas diferenciados, mediante su agrupación en " $n$ " factores, relativos a los componentes fundamentales utilizados comúnmente en el diseño de productos. El método persigue establecer la trazabilidad del diseño, determinando los constructos principales relativos a la función, ergonomía y forma de los productos , y todo ello orientado a la mejora en la definición conceptual de éstos, buscando en todo momento en lo que se refiere a gestión del conocimiento, la mejora en la aplicación de métodos, técnicas y procedimientos con objeto de optimizar el diseño y/o rediseño de los productos industriales.
\end{abstract}

Palabras Clave: Análisis Factorial, Diseño de Producto, Metodología del Diseño, Trazabilidad del diseño 


\begin{abstract}
It is intended to determine importance degree of the factors to be considered in the design of a product, from the considerations set out by both the consumer and designer perspective. By applying the method of Principal Component Analysis at the study of a case, we analyzed the desirable attributes for a product, in the opinion of experts and consumers. It is checked after the application of the method the existence of subsystems that explain the need to consider certain attributes (variables of the system), taking into account their degree of participation and affinity differentiated subsystems, by grouping them in " $n$ " factors relating to fundamental components commonly used in product design. The method aims to establish design traceability, determining the main constructs on the function, ergonomics and form of the products, all aimed at improving the conceptual definition of the product, always seeking in regard to knowledge management, improvement in the application of methods, techniques and procedures in order to optimize the design and / or redesign of industrial products.
\end{abstract}

Keywords: Factorial, Design Analysis Product, Design Methodology, Design Traceability

\title{
1. Introducción
}

Las discrepancias detectadas en investigaciones pasadas (CABELLO, 2009) entre consumidores y expertos en cuanto a las características y atributos que debe de tener en cuenta el diseño de un producto, así cómo, las diferencias detectadas desde las perspectivas de sus posiciones, como consumidores de productos industriales o teóricos conocedores de los requerimientos que estos deben poseer, nos hace reflexionar en el análisis de esta problemática y su repercusión e importancia a la hora de diseñar.

Desde la antigüedad encontramos que el análisis de productos con objeto de determinar sus factores integrantes, se ha realizado de manera más ó menos intuitiva. Nos consta que Vitrubio ya infiere una división de sus características en el 32 a.C. en estética, funcionalidad y reúso (LE DANTEC \& YI-LUEN DO, 2009). Tal vez esta sea la primera división de los factores integrantes del diseño. En el siglo pasado (MINTZBERG ,1991) comenta entorno a la forma, ergonomía y función. Actualmente y como consecuencia de la implementación de muchos modelos sistémicos podemos inferir patrones de recurrencia en esta división que prevén nuevas formas de trabajo, orientadas a una mejor definición del Diseño Conceptual (BRIEDE Y HERNANDIS, 2011).

Proponemos su análisis bajo la descomposición de los subsistemas fundamentales del diseño: forma, función y ergonomía considerando su isomorfismos en volúmenes, superficies y límites de contorno (Hernandis y Briede, 2009). Desde esta óptica del diseño analizaremos las características de los productos y estudiaremos mediante un caso propuesto cual es el resultado de esta investigación.

Es evidente que existen múltiples clasificaciones de los atributos del diseño que no se abordan en este trabajo, el motivo es evidente, al considerar que podría aplicarse el método a cualquier división pero su efectividad se ve enormemente mermada en caso de existir gran número de variables que se agrupan en muchos factores a su vez. Así mismo, también se han descartado otras metodologías de análisis (PAGE ET AL, 2001) por ser poco apropiadas según el objetivo planteado. 
Los estudios sobre los factores o características del producto son habituales tanto en la investigación de mercado como en lo relativo a innovación en desarrollo de nuevos productos (MALHORTA, 2004).

\section{Materiales y métodos}

\subsection{Metodología para el muestreo.}

La metodología utilizada Análisis de Componentes Principales (ACP) (SIOTANI Y WAKAKI, 2006) fue realizada bajo una base de Estadística cuantitativa, descriptiva, con entrevistas personales, por medio de un cuestionario que se creó durante el curso 2008-2009 entre alumnos que cursaban el Master de diseño gestión y desarrollo de nuevos productos de la Universidad Politécnica de Valencia, teniendo en cuenta aspectos relativos a la gestión del conocimiento para el diseño conceptual de productos industriales (ULUSOY, 1999). Se optó mediante una decisión consensuada, por estudiar un caso en relación a un producto conocido por gran número de usuarios, siendo elegido por los participantes un carro doméstico para la compra.

Se hizo un estudio pre-test, que tuvo como consecuencia el cambio en algunas de las preguntas del cuestionario, así como en el formato, que facilitaba una mejor comprensión del mismo (MALHORTA, 2004). Ya que se iba a realizar la encuesta por muchos encuestadores, esto disminuiría el error cometido por equivocaciones de interpretación del cuestionario.

Dichas entrevistas fueron realizadas en dos fases, en la primera se realizaron encuestas a personas que utilizaban el carro de compra doméstico, de manera habitual o incluso esporádica. La segunda fase se realizó con una muestra totalmente diferente, alumnos del Master oficial de Ingeniería del Diseño, así como a diferentes expertos en diversos ámbitos del diseño (BALL ORMEROD Y MORLEY, 2004). Se pretende que la muestra esté suficientemente diferenciada como para realizar diversos análisis comparativos que nos ayuden a responder a nuestros objetivos, así como a crear una base de datos para estudios posteriores.

El cuestionario consta de 26 preguntas en donde se hace referencia a la actitud, comportamiento y percepción del consumidor frente a diversos atributos generales (LE DANTEC Y YI-LUEN DO, 2009) de un carro de la compra, así como preguntas de clasificación general.

Para determinar el tamaño muestral adecuado hemos supuesto las siguientes hipótesis (MARTÍNEZ ET AL, 2000): la población de 800.666 habitantes, correspondiente a la población de Valencia. Si tenemos en cuenta tan solo la población útil para nuestro estudio (población entre 20 y 76 años de edad), esta se conforma por 410.068 lo que significa casi el $52 \%$ de la población total. Para efectos estadísticos consideramos la población como infinita, con un error de estimación del $5 \%$ y nivel de confianza del $90 \%$. Con estos datos, y aplicando la fórmula para intervalo de proporciones (Figura 1), hemos obtenido que el nivel adecuado de encuestas debe ser de 211.

\begin{tabular}{|c|c|}
\hline$n=Z_{\alpha / 2}^{2} \underline{p^{*} q}$ & $n=1,45^{2} \quad \underline{0,5^{2}}=211$ \\
\hline$\varepsilon^{2}$ & $0,05^{2}$ \\
\hline
\end{tabular}

Fig.1 Tamaño de la muestral 


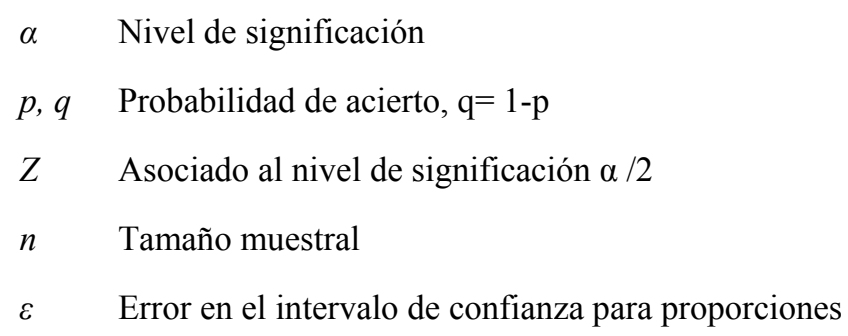

Se realizó un muestreo no probabilístico por conveniencia (AAKER ET AL, 2001), de las cuales 100 han sido realizadas a consumidores y el resto realizadas tanto a profesores como a estudiantes de diseño de la Escuela Técnica Superior de Ingeniería del Diseño, de la Universidad Politécnica de Valencia, así como a diseñadores con reconocida trayectoria profesional.

El primer estudio, viene dado por el tipo de usuarios de los que se requiere información, para ello se hizo un muestreo por conveniencia enfocado en individuos entre 20 y 76 años de edad residentes en Valencia capital captados de forma mayoritaria a la entrada de supermercados de alimentación, por ser estos los lugares más óptimos para localizar nuestro público objetivo

Los entrevistadores fueron nueve alumnos pertenecientes al Master en Diseño, Gestión y Desarrollo de Nuevos Productos de la Universidad Politécnica de Valencia, en su edición décimo segunda impartida durante el curso 2008-2009 y por tanto, perfectos conocedores de la investigación siendo estos quienes encuestaron a los consumidores.

\subsection{Análisis de los datos}

Para el análisis de los datos, hemos utilizado el programa informático SPSS 15.17. Se han realizado análisis de frecuencias para el estudio de la caracterización de la muestra. Es interesante contrastar si es aceptable la hipótesis de que las medias de todos los grupos de observaciones obtenidas al repetir el experimento para cada nivel de factor son idénticas. Si los contrastes diesen como resultado que esta hipótesis es cierta, la pertenencia a un grupo o a otro sería irrelevante, y podríamos considerar todas las observaciones como una muestra de una única población. Un enfoque alternativo de esta hipótesis, que conduce al mismo resultado, es considerar los grupos idénticos si las diferencias entre sus medias son pequeñas. El análisis de la varianza simple es una técnica estadística utilizada para analizar la relación entre una variable dependiente (o endógena) métrica y una o varias variables independientes (o exógenas no métricas). El objetivo esencial de los modelos del análisis de la varianza es determinar si diversas muestras proceden de poblaciones con igual media. De modo que el modelo ANOVA mide la significación estadística de las diferencias entre las medias de los grupos determinados en la variable dependiente por los valores de las variables independientes (ROMERO Y ZÚNICA, 2005).

Por otra parte para el estudio de nuestro objetivo, al observar muchas variables sobre una muestra, se decidió utilizar algún método multivariante de reducción de la dimensión. Estos métodos combinan muchas variables observadas para obtener pocas variables ficticias que las representen con la mínima pérdida de información.

Si son variables cuantitativas, las técnicas de reducción de la dimensión pueden ser el Análisis de Componentes Principales y el Análisis factorial, si son variables cualitativas, puede acudirse al Análisis de Correspondencias y al Escalamiento Óptimo, y si son variables cualitativas ordinales se acude al Escalamiento Multidimensional (PÉREZ-LÓPEZ, 2005). 
Teniendo en cuenta que las variables que se manejan son cuantitativas, y que ninguna de ellas se considera a priori dependiente principal de las demás, la técnica de reducción de la dimensión utilizada es el Análisis Factorial.

Dentro de esta técnica, se utilizó como método de extracción de los factores el método de los componentes principales, ya que SPSS considera este último como un caso particular del Análisis Factorial, utilizado para formar combinaciones lineales no correlacionadas de las variables observadas. Con este método, el primer factor será el que tenga una mayor varianza explicada, las componentes sucesivas explican menos varianza progresivamente, y no estarán correlacionadas entre ellas. Esto nos permite obtener una solución factorial inicial, así como la elección del número de factores más adecuado, para ello se tendrá en cuenta la varianza acumulada explicada por los primeros auto valores.

La finalidad del análisis factorial, es tener una interpretación clara de los factores, aunque esto no siempre es fácil, para ello se ha realizado una rotación de los factores a partir de la solución inicial, el método utilizado ha sido una rotación ortogonal por el método Varimax, que minimiza el número de variables que tienen saturaciones altas en cada factor.

\section{Resultados}

\subsection{Caracterización muestral}

En este apartado nos centramos en todas las características de la muestra objeto de nuestra investigación. Para ello hemos hecho un análisis de frecuencias de las variables referentes a: Género; Nacionalidad; Forma de vida y Nivel de Estudios; de los 211 encuestados, según

Tabla 1: Clasificación de la muestra

\begin{tabular}{|l|l|l|}
\hline \multirow{2}{*}{ GÉNERO } & hombre & $39,82 \%$ \\
\cline { 2 - 3 } & mujer & $60,18 \%$ \\
\hline \multirow{4}{*}{ ESTUDIOS } & sin bachiller & $15,18 \%$ \\
\cline { 2 - 3 } & bachiller & $29,46 \%$ \\
\cline { 2 - 3 } & diplomado & $23,21 \%$ \\
\cline { 2 - 3 } & licenciado & $32,14 \%$ \\
\hline
\end{tabular}

\begin{tabular}{|l|l|l|}
\hline \multirow{2}{*}{ NACIONALIDAD } & español & $84,17 \%$ \\
\cline { 2 - 3 } & extranjero & $15,83 \%$ \\
\hline \multirow{3}{*}{ FORMA DE VIDA } & solo & $15,93 \%$ \\
\cline { 2 - 3 } & pareja & $13,27 \%$ \\
\cline { 2 - 3 } & familia o comparte & $70,80 \%$ \\
\hline
\end{tabular}

Como podemos apreciar la distribución de los encuestados en cuanto al Género hay un porcentaje mayor de mujeres que usan habitualmente el carro de compra. Mientras que los hombres encuestados representan sobre el $40 \%$, las mujeres representan el $60 \%$. Este es un dato que no nos sorprende, aunque presuponemos que irá igualándose en el tiempo, dada la evolución del papel de la mujer en la sociedad española.

Mayoritariamente nuestros encuestados son de nacionalidad española, la muestra en este sentido, ha sido seleccionada de forma aleatoria, por lo que podemos aproximar que la población está distribuida de la misma manera, con un casi $20 \%$ de extranjeros. 
Hay que tener en cuenta que nuestra muestra no es aleatoria, ya que gran parte de ella se ha realizado entre alumnos de la Universidad Politécnica de Valencia, que además en su mayoría estaban realizando estudios de postgrado, por lo que más del $50 \%$ de los encuestados, tenían como mínimo una diplomatura realizada. Aún así se realizó el análisis de la muestra sin tener en cuenta las encuestas realizadas en la Universidad, dejando patente que seguía existiendo muy pocas personas que no tuvieran como mínimo estudios de graduación secundaria.

Vemos como mayoritariamente, se vive con la familia o se comparte piso con compañeros, por el origen de la muestra, (principalmente estudiantes de diseño), y la edad de los encuestados, parece bastante natural este resultado.

\subsection{Comparación de medias}

Comparamos las medias sobre la opinión respecto a los atributos del producto entre alumnos de diseño y los consumidores. Tomamos como variable independiente los alumnos de diseño, y como variable exógena los consumidores. Para determinar si existe verdaderamente diferencia entre las medias, aplicamos un análisis ANOVA para un factor.

Resumimos en la Tabla II los factores que por tener una significación menor al 10\%, se puede rechazar la hipótesis de igualdad entre sus medias, y facilitar de esta manera la interpretación visual de los datos. Presenta las sumas de los cuadrados para cada fuente de variación, los cuadrados medios, el valor de la $\mathrm{F}$ de Fisher-Snedecor para el contraste global de diferencias significativas entre todas las medias de cada nivel de factor y los p-valores, que permite decidir entre aceptar o rechazar la diferencia significativa entre medias de cada nivel de factor. Si el p-valor resulta menor a 0,1 se acepta que las medias de las muestras para cada nivel de factor difieren significativamente al $90 \%$. Por tanto serán estos valores los únicos dignos de estudio posterior. En la tabla también se muestran las medias de valoración para cada uno de los atributos según cada tipo de encuestado, en los que inferimos que la opinión de los estudiantes es distinta a la del resto de consumidores.

Tabla 2: Anova

\begin{tabular}{|c|c|c|c|c|c|c|c|}
\hline ANOVA & Suma de cuadrados & Media cuadrática & $\mathbf{F}$ & Sig. & $\begin{array}{l}\text { Alumnos } \\
\text { de diseño }\end{array}$ & consumidor & $\begin{array}{l}\text { Media } \\
\text { totales }\end{array}$ \\
\hline precio & 3,731 & 3,731 & 3,524 & 0,063 & 2,68 & 3,05 & 2,92 \\
\hline impermeable & 2,161 & 2,161 & 2,182 & 0,142 & 2,73 & 3,01 & 2,91 \\
\hline limpieza interior & 3,919 & 3,919 & 3,647 & 0,059 & 2,63 & 3,01 & 2,88 \\
\hline limpieza exterior & 4,901 & 4,901 & 5,043 & 0,027 & 2,53 & 2,96 & 2,81 \\
\hline acoplable a bici & 4,707 & 4,707 & 3,893 & 0,051 & 2,31 & 1,88 & 2,03 \\
\hline
\end{tabular}

Los alumnos le dan menos importancia al precio, a que sea impermeable, la limpieza interior y exterior, y sin embargo valoran más que el resto de consumidores que sea acoplable a una bicicleta.

\subsection{Análisis de componentes principales}

El determinante de la matriz de correlaciones nos da un valor de 3,984E-06, por ser muy pequeño, nos indica que la condición inicial para el análisis de las componentes principales es adecuada, ya que demuestra que el grado de intercorrelación entre las variables es muy alto. Así mimo, la correlación con 
respecto a los factores la confirma el test de esfericidad de Barlett. Como su valor p-valor es 0,000 , se puede concluir que existe correlación significativa con las variables.

Observamos también en la Tabla 3 el estadístico KMO, cuyo valor 0,740 muy por encima de 0,5 nos asegura una muy buena adecuación de la muestra a este análisis.

Tabla 3: KMO y prueba de Bartlett

\begin{tabular}{|l|l|c|}
\hline \multicolumn{2}{|l|}{ Medida de adecuación muestral de Kaiser-Meyer-Olkin. } &, 740 \\
\hline Prueba de esfericidad de Bartlett & Chi-cuadrado aproximado & 1241,260 \\
\hline & gl & 378 \\
\hline & Sig. &, 000 \\
\hline
\end{tabular}

Analizamos a continuación la comunalidad de las variables (suma de los cuadrados de sus cargas factoriales definidas en la matriz de componentes), comprobando así la variabilidad de cada atributo que es explicada por los factores. Observamos que la comunalidad de todas las variables después de las extracciones es superior a 0.3 , excepto la referida a tener departamento para frágiles y a que sea plegable, que tienen un valor inferior.

Retenemos 4 de los 7 factores que tienen una varianza explicada mayor que uno, ya que estos son los que explican la mayor parte de la varianza, como podemos comprobar en la Tabla 4 de autovalores iniciales. Quedaría explicada el $47,27 \%$ de la varianza total.

Tabla 4: Varianza total explicada

\begin{tabular}{|c|c|l|l|}
\hline Componente & \multicolumn{3}{|l|}{ Autovalores iniciales } \\
\hline & Total & \% de la varianza & \% acumulado \\
\hline 1 & 6,24 & 22,29 & 22,29 \\
\hline 2 & 3,14 & 11,23 & 33,51 \\
\hline 3 & 1,97 & 7,04 & 40,55 \\
\hline 4 & 1,88 & 6,73 & 47,27 \\
\hline
\end{tabular}

Por lo que obtenemos la matriz de componentes. Utilizamos la Tabla 5 de componentes rotados para facilitar enormemente la claridad de pertenencia de cada una de las variables a un componente, el método utilizado para la rotación ha sido una rotación Varimax, ya que tiene la propiedad de que los factores siguen siendo incorrelados. Las celdas vacías corresponden a coeficientes menores de 0,2 que no aparecen en la tabla para facilitar visualmente la interpretación de los coeficientes representativos. 
Tabla 5: Matriz de componentes rotados.

\begin{tabular}{|l|l|l|l|l|}
\hline & 1 & 2 & 3 & 4 \\
\hline estética & & 0,75 & & \\
\hline accesibilidad interior & 0,60 & & & \\
\hline escaleras & 0,57 & & & \\
\hline manejabilidad & 0,74 & & & \\
\hline peso carga & 0,62 & & & \\
\hline peso vacio & 0,45 & 0,24 & & 0,32 \\
\hline volumen carga & 0,38 & & & 0,41 \\
\hline congelados & 0,20 & & 0,61 & \\
\hline frágiles & & & 0,44 & \\
\hline precio & 0,42 & 0,38 & $-0,35$ & $-0,22$ \\
\hline maletero con compra & & & 0,23 & 0,78 \\
\hline maletero en vacío & & & & 0,78 \\
\hline guardar en casa & 0,54 & 0,47 & $-0,25$ & \\
\hline impermeable & & & & $-0,36$ \\
\hline
\end{tabular}

\begin{tabular}{|l|l|l|l|l|}
\hline & 1 & 2 & 3 & 4 \\
\hline limpieza interior & 0,47 & 0,47 & 0,23 & \\
\hline limpieza exterior & 0,26 & 0,41 & 0,28 & $\begin{array}{l}- \\
\end{array}$ \\
\hline lavadora & & 0,36 & 0,52 & - \\
\hline otros usos & & & 0,60 & \\
\hline desmontable & 0,23 & 0,20 & 0,43 & \\
\hline manejo en la conducción & 0,68 & 0,20 & & \\
\hline comodidad agarre & 0,73 & & 0,23 & \\
\hline regulable altura & 0,57 & & 0,33 & \\
\hline regulable volumen & 0,53 & & 0,35 & 0,25 \\
\hline acoplable a bici & & & 0,69 & \\
\hline plegable & & & 0,30 & \\
\hline moderno & & 0,80 & & \\
\hline diseño interior & & 0,61 & 0,36 & \\
\hline diseño exterior & & 0,76 & & \\
\hline
\end{tabular}

\subsection{Consulta a expertos}

Con el fin de poder en un principio interpretar los cuatro factores retenidos en primera instancia con el análisis de componentes principales, se consulta a 6 expertos en diseño, procedentes de diferentes países, ya que de esta manera se contrastan diferentes opiniones según áreas de especialización heterogéneas y diferentes entre si. Como resultado de esta puesta en común de opiniones, refutamos la clasificación determinada por los conjuntos de variables determinados como FORMA, FUNCIÓN Y ERGONOMÍA sin caer en posibles subjetividades que podrían haberse creado con grupos u orígenes más homogéneos.

Cada uno de los factores implica una agrupación de características o atributos del diseño que intentamos identificar con la propuestas realizadas por autores representativos. En particular nos centraremos en la propuesta de Mintzberg que propone una división en Forma, Función y Ergonomía y que en la actualidad viene a ser una de las más utilizadas en las Escuelas de Diseño. En particular en la ETSID y dentro de los denominados Métodos Sistémicos (HERNANDIS, 2000) es una división habitual.

Consultados diversos especialistas con respecto a cómo las características se agrupan según los factores mencionados, proponemos identificar el Factor 1 con Ergonomía. Del mismo modo se concluye que los atributos del Factor 2 se identifican con Forma. Y de igual modo el Factor 3 se identifica con aspectos de 
la Función. Por último en el Factor 4 aparecen dos características aisladas, cuya explicación en este caso se debe a que implica a otro producto, siendo su existencia necesaria para que tenga sentido el producto complementario (vehículo). Suponemos por tanto que se refiere a accesorios. En la siguiente Tabla 6 se resume esta asignación.

Tabla 6: Atributos de Diseño

\begin{tabular}{|c|c|c|c|}
\hline $\begin{array}{l}\text { Factor 1: } \\
\text { ERGONOMÍA }\end{array}$ & $\begin{array}{l}\text { Accesibilidad interior } \\
\text { Que suba escaleras } \\
\text { Manejabilidad } \\
\text { Peso que soporta } \\
\text { Peso del carro en vacío } \\
\text { Precio } \\
\text { Fácil de guardar en casa } \\
\text { Impermeable } \\
\text { Manejo en la conducción } \\
\text { Comodidad de agarre } \\
\text { Regulable en altura } \\
\text { Regulable volumen }\end{array}$ & $\begin{array}{l}\text { Factor 2: } \\
\text { FORMA }\end{array}$ & $\begin{array}{l}\text { Estética } \\
\text { Fácil de limpiar por fuera } \\
\text { Moderno } \\
\text { Diseño interior } \\
\text { Diseño exterior }\end{array}$ \\
\hline Factor 3: FUNCIÓN & $\begin{array}{l}\text { Apartado congelados } \\
\text { Apartado para frágiles } \\
\text { Lavable en lavadora } \\
\text { Posibilidad de otros usos } \\
\text { Desmontable } \\
\text { Acoplable a una bici } \\
\text { Plegable }\end{array}$ & $\begin{array}{l}\text { Factor 4: } \\
\text { ACCESORIOS }\end{array}$ & $\begin{array}{l}\text { Fácil de meter en el maletero con compra } \\
\text { Fácil de meter en el maletero vacío }\end{array}$ \\
\hline
\end{tabular}

Es evidente la identificación de atributos según los especialistas consultados y su agrupamiento en los sub-sistemas forma, función y ergonomía.

\section{Conclusiones}

Esto posibilita el análisis de correspondencia entre los factores de diseño y la opinión del consumidor y diseñador con las características de éste, es decir, pretende ser un primer paso entre lo que denominaremos trazabilidad del diseño. Se pretende en futuras investigaciones buscar la máxima correlación entre los atributos, especificaciones o características que afectan al diseño de producto, las características demandadas por los consumidores y el conocimiento del diseñador como alquimista del diseño. El agrupamiento de los factores, sus posibles sub-agrupamientos en subsistemas fundamentales y el estudio de las relaciones existentes en razón de sus propiedades y comportamientos según la funcionalidad, ergonomía y forma, hacen prever un camino interesante para el uso del Análisis de Componentes Principales con objeto de determinar la trazabilidad de diseño. 


\section{Referencias}

AAKER, D.A., KUMAR, V \& DAY, G. (2001). Investigación de mercados, México: Limusa Wiley.

BALL, L.J, ORMEROD, T.C \& MORLEY, N.J. (2004). Spontaneous analogising in engineering design: a comparative analysis of experts and novices. Design Studies 25, 495-508.

BRIEDE, J.C \& HERNANDIS, B. (2011). New methods in design education: The systemic methodology and the use of sketch in the conceptual design stage. US-China Education Review, 8 (1), 118-128.

CABELLO, M. (2009). Estudio de los factores de diseño gráfico de la etiqueta de vino tinto de calidad que influyen en su elección y compra: estudio cuantitativo en valencia. PhD Thesis, Valencia: Universidad Politécnica de Valencia.

HERNANDIS, B. (2003). Desarrollo de una metodología sistémica para el diseño de productos industriales. $\mathrm{PhD}$ Thesis, Valencia: Universidad Politécnica de Valencia.

HERNANDIS, B. \&, BRIEDE, J.C. (2009). An educational application for a product design and engineering systems using integrated conceptual models. Ingeniare, 17 (3), 432-442.

LE DANTEC, C.A. \& YI-LUEN, D.O. E. (2009). The mechanisms of value transfer in design meetingsThe mechanisms of value transfer in design meetings. Design Studies, 30, 119-137.

MALHORTA, N.K. (2004). Investigación de Mercados. México: Pearson Educación.

MARTÍNEZ, J. ET AL. (2000). La investigación en marketing. Barcelona: Aedemo.

MINTZBERG H (1991). Managing the Form, Function and Fit of Design. Ed. Design Management Journal, 2(3).

PÉREZ-LÓPEZ, C. (2005). Métodos estadísticos avanzados con SPSS. Madrid: Thomson.

ROMERO, R. \& ZÚNICA, L. (2005). Métodos estadísticos en ingeniería. Valencia: Universidad Politécnica de Valencia.

PAGE, A., PORCAR, R., SUCH, M.J., SOLAZ, J. \& BLASCO, V. (2001). Nuevas técnicas para el desarrollo de productos innovadores orientados al usuario. Valencia: Instituto de Biomecánica de Valencia y ADCV

SIOTANI, M. \& WAKAKI, H. (2006). Contributions to multivariate analysis by Professor Yasunori Fujikoshi. Journal of Multivariate Analysis, 97, 1914 - 1926.

ULUSOY, Z. (1999) .To design versus to understand design: the role of graphic representations and verbal expressions. Design Studies 20, 123-130. 